\title{
The Distinction of Licheniformin from Subtilin by Cross- Reactions with Antibiotic-Resistant Strains of Mycobacterium phlei
}

\author{
BY P. D'ARCY HART AND BRENDA MOSS \\ The National Institute for Medical Research, Mill Hill, London, N.W. 7
}

\begin{abstract}
SUMMARY: Under standard conditions of subculturing in increasing drug concentrations, licheniformin and subtilin (polypeptide-containing antibiotics from the genus Bacillus) induced resistance in Mycobacterium phlei more slowly and to a much smaller degree than did streptomycin. Strains resistant to licheniformin and subtilin tended to revert, whereas streptomycin-resistance was stable. Licheniformin resistance was somewhat more difficult to induce and less stable than subtilin resistance.

Cross-resistance tests of bacteriostatic activity between the resistant strains and the three antibiotics distinguished completely licheniformin from both subtilin and streptomycin. Although the difference between licheniformin and subtilin was largely confirmed by a more sensitive bactericidal test, making viable counts after varying periods of exposure of the resistant strains to the antibiotics in nutrient medium, the test, nevertheless, revealed a minor overlap between the two antibiotics, which was either the result of contamination of one by the other or, more probably, the expression of some common chemical property. Licheniformin and subtilin differed in the speed of their lethal action on $M$. phlei, subtilin being much the slower.

When M.phlei was exposed to mixtures of streptomycin and licheniformin in certain proportions, the rapid development of high streptomycin resistance was prevented.
\end{abstract}

Under standard conditions, it is possible to compare the rates of emergence in vitro of bacterial strains resistant to different antibacterial substances by repeated subculture of an initially sensitive strain of a suitable bacterium in graded concentrations of these substances. Such resistant cultures can be used for cross-resistance tests, and so serve as a tool for the study of relations between antibacterial substances.

This method was successfully applied to Streptococcus pyogenes and Staphylococcus pyogenes and various synthetic drugs, including sulphonamides, diamidines and acridines (McIntosh \& Selbie, 1943; Wien, Harrison \& Freeman, 1948), and to antibiotics like streptomycin and streptothricin, using induced resistant strains of Staph. pyogenes (Sullivan, Stahly, Birkeland \& Myers, 1946) or Mycobacterium tuberculosis (Smith \& Waksman, 1947). Schemes for identifying new antibiotics by means of standard resistant strains have been proposed by Eisman, Marsh \& Mayer (1946) and by Stansly (1948).

We felt that subtilin (Jansen \& Hirschmann, 1944; Lewis, 1947) and licheniformin (Callow, Glover, Hart \& Hills, 1947) could be profitably examined by this method, because although distinguished by certain physical and chemical properties, as well as by their range of antibacterial activity, they show some interesting similarities, such as origin from species of the genus Bacillus (B. subtilis and B. licheniformis), basic nature and polypeptide composition, relative thermostability at low pH's, and activity against mycobacteria, including Mycobacterium tuberculosis. Streptomycin was also tested to widen the comparison. $M$. phlei was used throughout. 
Three main methods were used: (1) measurement of the rate and degree of development of resistant strains to the three antibiotics, and of-the stability of these strains; (2) tests of cross-resistance to bacteriostasis; and (3) tests of cross-resistance to killing.

\section{MATERIALS AND METHODS}

Antibiotics used. The licheniformin used, containing a mixture of polypeptides of differing activities against mycobacteria, and some inactive impurities (Callow \& Work, 1949), was batches 551 and 855, with potencies 5000 and 10,000 units/mg. (Callow et al. 1947). Solutions were prepared by dissolving in dil. $\mathrm{HCl}$ at $\mathrm{pH} 2.5$ and autoclaving at $10 \mathrm{lb} . / \mathrm{in} .^{2}$ for $10 \mathrm{~min}$. The subtilin, about $90 \%$ pure (Lewis, 1949), was lot 317 , with a potency $2 \cdot 3$ times that of the standard (Fevold, Dimick \& Klose, 1948) (in the absence of an agreed unitage, weights are recorded below); solutions were made as for licheniformin and heated for 5 min. in a boiling water bath. The streptomycin was a commercial preparation of the sulphate, potency $900 \mathrm{units} / \mathrm{mg}$. (i.e. c. $900 \mu \mathrm{g}$. base/mg.); solutions in distilled water were treated as sterile.

The test bacterium. M. phlei was preferred to a virulent $M$. tuberculosis because of its more rapid growth and greater safety. A laboratory strain, $\mathbf{P}$, previously used for routine assays of licheniformin, was chosen. This strain was also sensitive to subtilin and to streptomycin. Both the original strain, to which the designation $P$ is restricted, and all the resistant strains derived from it, were maintained on slopes of Herrold's (1981) egg-yolk agar medium, containing $5 \%$ glycerol. For bacteriostatic tests, suspensions were prepared by grinding the 4-6 days' growth from this medium in Griffith tubes and suspending in distilled water at $0 \cdot 2 \mathrm{mg}$. (moist weight)/ml., as judged by opacity comparisons with a standard; such suspensions contained about $25 \times 10^{7}$ viable organisms $/ \mathrm{ml}$. The inoculum for $5.5 \mathrm{ml}$. of the Dubos medium (see below) was $0.2 \mathrm{ml} .=0.04 \mathrm{mg}$. moist weight, or $c .5 \times 10^{7}$ viable organisms. For bactericidal tests, in which periodic viable counts were made, 5.5 ml. of Dubos medium, inoculated as above with $0.04 \mathrm{mg}$. bacilli, were incubated at $37^{\circ}$ for 3 days; the culture was then centrifuged lightly to bring down large clumps, and the upper part of the suspension used. The inoculum was $2.0 \mathrm{ml}$. for flasks of $55 \mathrm{ml}$. of the Dubos medium containing the different antibiotics; the resulting mixtures usually contained c. $10^{6}$ viable organisms $/ \mathrm{ml}$.

\section{Production of resistant strains}

In liquid media. The repeated subculture of $M$. phlei $\mathbf{P}$ in Hartley's digest broth containing graded concentrations of antibiotic, as was done with subtilin by Salle \& Jann (1948), proved unsatisfactory owing to uneven granular growth, particularly near the inhibition end-point concentration. Tweenalbumin medium, essentially that of Dubos \& Davis (1946), was used thereafter. The basal part of this medium was Difco Casamino acids, 2.5 g.; asparagine, 0.3 g.; anhydrous $\mathrm{Na}_{2} \mathrm{HPO}_{4}, 2.5 \mathrm{~g}$.; $\mathrm{KH}_{2} \mathrm{PO}_{4}, 1.0$ g.; sodium citrate, 1.5 g.; $\mathrm{MgSO}_{4}, 7 \mathrm{H}_{2} \mathrm{O}, 0.6 \mathrm{~g}$.; glycerol, $25 \mathrm{ml}$; distilled water to $1 \mathrm{l}$. It was distributed in $5 \mathrm{ml}$. quantities in $25 \mathrm{~mm}$. diameter test tubes and autoclaved. 
To each tube $0.25 \mathrm{ml}$. of $5 \%$ albumin (bovine plasma fraction $\mathrm{V}$ ), filtered through Ford Sterimat SB or through Gradocol membrane $0.56-0.9 \mu$., and $0.25 \mathrm{ml}$. of $1 \%$ Tween 80 , autoclaved at $15 \mathrm{lb} . / \mathrm{in} .{ }^{2}$ for $15 \mathrm{~min}$., were added aseptically, making a final volume of $5.5 \mathrm{ml}$. A standard suspension of strain $\mathbf{P}$ was inoculated (in $0.04 \mathrm{mg}$. quantities) into tubes of this medium containing serial two-fold dilutions of antibiotic, and the lowest concentration giving complete inhibition of growth after 8-6 days' incubation at $37^{\circ}$ was taken as end-point of the initial bacteriostatic sensitivity assay. Samples $(0.2 \mathrm{ml}$. $)$ from the tube with the highest concentration of antibiotic permitting definite visible growth were seeded into a new set of tubes, which were incubated, and the process repeated. In later experiments each transfer was made by way of egg-yolk agar and the inocula obtained from this intermediate culture. Thus, when the egg medium culture, grown from a loopful from the appropriate tube, and representing strain $\mathbf{P}$ modified by the first exposure to a certain concentration of antibiotic, was 4-6 days old, a standard suspension made from it was again seeded into dilutions of the antibiotic to test sensitivity and to provide the next subculture on egg for the third exposure. The process of transfer and assay was repeated as many times as desired. By this means, the direct transfer of unknown and variable inocula from one series to the next was avoided; the inocula were approximately standard and the sensitivity assays comparable.

On solid medium. The dried surfaces of $9 \mathrm{~cm}$. plates of Hartley's digest broth agar, containing serial dilutions of antibiotic, were seeded uniformly with $0.2-0.5 \mathrm{ml}$. of a heavy suspension of strain $\mathbf{P}$ from egg medium. The plates were sealed, inverted and incubated at $37^{\circ}$ for 4-10 days, until good growth was obtained on a control plate. Colonies from the plate containing the highest concentration of antibiotic were subcultured on egg-medium slopes, and the process was repeated.

\section{Bacteriostatic cross-resistance tests}

Sets of tubes of Dubos medium (5.5 ml.), containing serial two-fold dilutions of the different antibiotics, received $0.2 \mathrm{ml}$. of standard suspensions $(0.04 \mathrm{mg}$. bacilli) of the different resistant strains and of strain $P$, and were incubated at $37^{\circ}$. Inhibitory end-points were read daily for 6 days.

\section{Bactericidal cross-resistance tests}

The Dubos basal medium was autoclaved in $50 \mathrm{ml}$. amounts in $250 \mathrm{ml}$. conical flasks and $2.5 \mathrm{ml}$. of sterile albumin and of Tween 80 then added. After introducing the antibiotics the flasks were inoculated with $2.0 \mathrm{ml}$. of suspensions (p. 245) of the strains under test and incubated at $37^{\circ}$; at intervals during $30 \mathrm{hr}$. the flasks were shaken and samples taken for viable counts.

For the counts, $9 \mathrm{~cm}$. plates, containing $15 \mathrm{ml}$. of $5 \%$ horse blood in Hartley's digest broth agar, were used. The dropping-pipette technique of Miles \& Misra (1938) was modified in two respects: (1) two separate drops of each dilution were inoculated on each of three plates, instead of one on each of six, the counts being averages of the six drops of a given dilution; (2) the dilutions of the test 
fluids containing the bacterial suspensions were made in distilled water instead of in medium or in normal saline; tests showed that over the period (maximum 30 min.) between making the dilutions and inoculating the plates, the lethal effect of water on $M$. phlei (P) was no different from that of saline, both being negligible. After 3-5 days at $37^{\circ}$, usually two of the ten-fold dilutions $\left(10^{-1}\right.$ $10^{-5}$ ) yielded easily countable colonies and the two counts agreed well. The presence of surface-active Tween 80, and in some cases of subtilin, in the test mixtures made the volume/drop of samples taken for dilution slightly less than that of water; conversion factors were calculated from measurements of these slight differences (see Fildes, 1931), and due allowance made in the readings.

The antibiotic in the fluids sampled did not unduly affect the fluids diluted for the plate-counts. Dubos medium containing 125 units licheniformin/ml. was diluted $\left(10^{\circ}\right.$ to $\left.10^{-4}\right)$ in water. To $1 \mathrm{ml}$. of each dilution, and to a drug-free control, was added $0.1 \mathrm{ml}$. of a 3-day culture of $M$. phlei $\mathbf{P}$ in Dubos medium, diluted to give a count of 50-100 organisms $/ 0.02 \mathrm{ml}$. drop in the control. Immediately each of three blood agar plates was inoculated with a drop of the mixtures. The same procedure was followed with a solution of $200 \mu \mathrm{g}$. subtilin/ ml. After 3 days' incubation, the average viable counts were:

\begin{tabular}{lcc} 
& \multicolumn{2}{c}{ Colonies/drop } \\
\cline { 2 - 3 } Dilution & Licheniformin & Subtilin \\
$10^{\circ}$ & 0 & 0 \\
$10^{-1}$ & 56 & 77 \\
$10^{-2}$ & 76 & 81 \\
$10^{-8}$ & 83 & 61 \\
$10^{-4}$ & 73 & 62 \\
Control & 62 & 75
\end{tabular}

That is, dilutions of $1^{-1}$ downwards gave counts approximately equal to those in drug-free controls. The lowest dilution of the test mixtures that could safely be used for plating was therefore clearly $10^{-1}$, and this was adopted as a routine.

\section{RESULTS}

\section{Production of resistant strains}

In Dubos medium, the lowest concentrations of the three different antibiotics completely inhibiting $M$. phlei $\mathbf{P}$ were 2 units licheniformin $/ \mathrm{ml}$, $3 \mu \mathrm{g}$. subtilin/ $\mathrm{ml}$. and 0.4 unit streptomycin/ml. Direct transfers were made into new sets of tubes; after twenty-two such transfers in licheniformin-containing medium, in a period of 5 months, the resulting strain was grown on egg medium and its sensitivity compared with that of the unmodified strain P; it was 60 units licheniformin/ml, or $1 / 30$ of the original value for $P$. Since this ratio was $1 / 2$ after three and $\mathbf{1 / 1 6}$ after eleven of the twenty-two transfers, the rate of decrease was clearly slow. In contrast, the sensitivity to subtilin in Dubos medium decreased to $1 / 30$, and to streptomycin to $1 / 5000$, of the initial values for strain $P$ after three transfers.

To improve comparability, the use of intermediate egg-yolk agar cultures as a stage in the transfers, with standardization of suspensions prepared therefrom 
for inoculation (p. 245), was adopted in a concurrent comparison of the rates of production of resistant strains in Dubos medium containing increasing antibiotic concentrations, starting again from strain $P$. Fig. 1 records the sensitivities of each of the strains after five exposures to the three antibiotics,

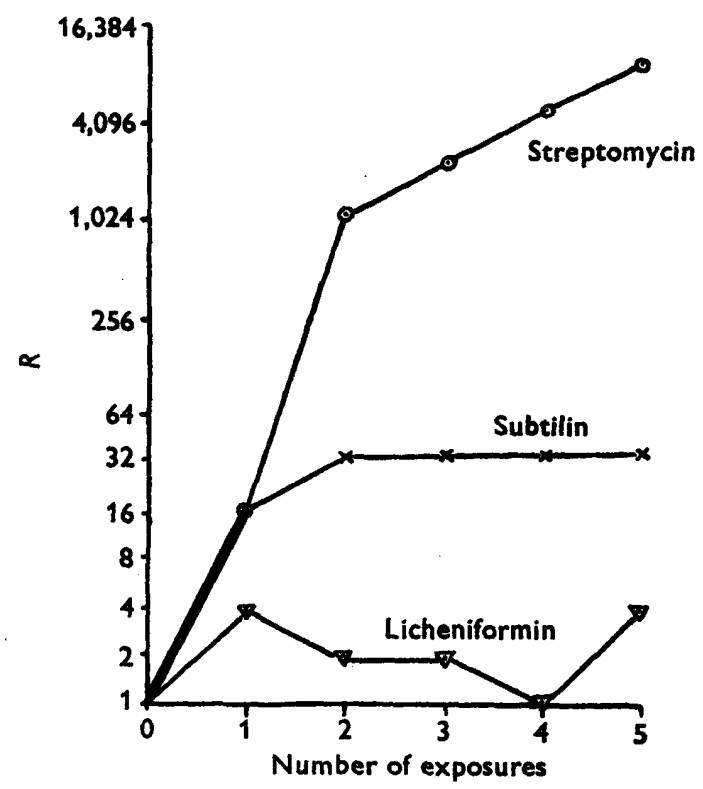

Fig. 1. Increase in resistance of $M$. phlei to licheniformin, subtilin and streptomycin, after repeated subculture in graded concentrations of antibiotic. $R=$ ratio of the minimum inhibiting concentrations of the antibiotics to the initial minimum inhibiting concentrations, which were licheniformin, 2 units/ml.; subtilin, $3 \mu \mathrm{g} . / \mathrm{ml}$.; and streptomycin, $\mathbf{0 \cdot 2}$ unit/ml.

expressed as inverse ratios to the corresponding initial sensitivities of the unmodified P. It is clear that streptomycin-resistance increases rapidly to its very high value of more than 10,000-fold, whereas licheniformin and subtilin resistance reach limits at levels not much above the initial values.

In case the feeble licheniformin resistance was due to the medium, attempts were made to induce resistance on agar plates. In the first set of plates there was no growth on agar containing 200 units licheniformin/ml., but 100 colonies on 100 units $/ \mathrm{ml}$. After eight repetitions a small amount of growth was visible on the plate containing 5000 units $/ \mathrm{ml}$. In a similar experiment with streptomycin, one colony grew on the plate containing 1000 units $/ \mathrm{ml}$., even in the first set.

It is more difficult to maintain on nutrient agar a bacterial population resistant to licheniformin than a population resistant to streptomycin. Licheniformin, subtilin and streptomycin were incorporated in $9 \mathrm{~cm}$. broth agar plates at concentrations designed to cover a similar range. One-half of the surface of each plate was uniformly seeded with a light suspension of $M$. phlei $(0.04 \mathrm{mg}$. moist weight) resistant to the given antibiotic, and the other half with a heavy suspension (0.4 mg.), and the plates were sealed and incubated. The suspensions 
were from egg-medium cultures of strains insensitive to 5000 units licheniformin $/ \mathrm{ml}$., 500 units streptomycin $/ \mathrm{ml}$. and $1000 \mu \mathrm{g}$. subtilin $/ \mathrm{ml}$., respectively. Table 1 shows the growth after 7 days. It needed a heavy inoculum of bacteria previously resistant to 5000 units licheniformin $/ \mathrm{ml}$. to establish growth at the same concentration, and even at 100 units/ml. growth was not profuse; light inocula failed to grow at the higher concentration and grew only slightly at the lower. With streptomycin-resistant bacteria, on the other hand, even a light inoculum grew as profusely at 1000 units $/ \mathrm{ml}$. as it did on the control plate.

Table 1. Growth from suspensions of $\mathrm{M}$. phlei previously resistant to licheniformin, subtilin and streptomycin, seeded on nutrient agar plates containing graded concentrations of the same respective antibiotics

Antibiotic to which $M$. phlei had been resistant, and against which it was re-tested

\begin{tabular}{|c|c|c|c|c|c|c|c|c|c|c|c|}
\hline \multirow{2}{*}{$\begin{array}{l}\text { Bac- } \\
\text { terial } \\
\text { uspen- } \\
\text { sion }\end{array}$} & \multicolumn{4}{|c|}{ Licheniformin } & \multicolumn{4}{|c|}{ Subtilin } & \multicolumn{3}{|c|}{ Streptomycin } \\
\hline & 6000 & 100 & $\begin{array}{c}10 \\
\text { units/ml. }\end{array}$ & $\mathbf{0}$ & 1000 & 10 & $\mu \mathrm{g} . / \mathrm{ml})$. & $\mathbf{0}$ & 1000 & $\begin{array}{ll}10 & 1 \\
\text { (units/ml.) }\end{array}$ & $\overrightarrow{0}$ \\
\hline ight & $\mathbf{0}$ & \pm & $+t+$ & +++ & \pm & +++ & +++ & +++ & $+t+$ & $+t+$ & $+t+$ \\
\hline Ieavy & Tr. & $t+$ & $t+t$ & +++ & + & +++ & +++ & +++ & +++ & $+t+t+t$ & $++t$ \\
\hline
\end{tabular}

Tr.,,,, \pm++++++ indicate degrees of growth.

The behaviour of subtilin-resistant organisms was intermediate. In the case of licheniformin, therefore, and to a lesser extent of subtilin, highly resistant strains were difficult to maintain and had partially reverted to sensitive, in sharp contrast to the stability of the streptomycin-resistant strain.

In another comparison of stability, resistant colonies from broth agar containing high concentrations of the three antibiotics were subcultured on egg medium, and standard suspensions re-tested against the same antibiotics in the Dubos medium. The licheniformin-resistant organisms now resisted only 8 units licheniformin $/ \mathrm{ml}$. instead of 5000 units $/ \mathrm{ml}$. previously in agar; the subtilin-resistant strain $64 \mu \mathrm{g}$. subtilin $/ \mathrm{ml}$. instead of $1000 \mu \mathrm{g} . / \mathrm{ml}$; ; but the streptomycin-resistant organisms were still resistant to 1000 units streptomycin/ml. The resistances in Dubos medium were 8, 40 and 10,000 times those of the unmodified strain $P$ tested at the same time. The contrast between resistance to licheniformin and to streptomycin, with subtilin resistance intermediate, is again evident.

\section{Cross-resistance tests}

Bacteriostatic test. The sensitivity of licheniformin-resistant, subtilin-resistant and streptomycin-resistant $M$. phlei (being the fifth transfers in the experiment illustrated in Fig. 1), as well as that of strain $\mathbf{P}$ from which they were derived, was measured simultaneously against each of the three antibiotics in Dubos medium. Table 2 gives the 3-day readings in one experiment. The resistance of a modified strain to an antibiotic is indicated by the ratio of the bacteriostatic titre of the antibiotic against this strain to its activity against the original, unmodified strain; a difference less than four-fold indicates 
Table 2. Minimal inhibiting concentrations* of three antibiotics for induced resistant strains of $\mathbf{M}$. phlei

Modified strains
Antibiotic

Streptomycin

Licheniformin

Subtilin

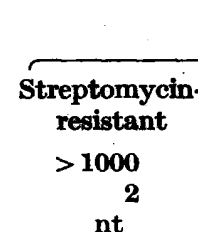

nt
* In units/ml. of Dubos liquid medium for streptomycin and licheniformin, and in $\mu \mathrm{g} . / \mathrm{ml}$. for subtilin. $n t=$ no test.

susceptibility and a four-fold or greater difference, resistance. The contrasts that then emerge are: (1) the highly streptomycin-resistant strain was susceptible to licheniformin, and the slightly licheniformin-resistant strain was susceptible to streptomycin; (2) the moderately subtilin-resistant strain was susceptible to licheniformin, and the slightly licheniformin-resistant strain was susceptible to subtilin. There is no evidence of cross-resistance or of overlapping activity, and on this basis licheniformin is distinguished both from streptomycin and from subtilin. The first of the two contrasts would be sharper if licheniformin resistance were of a higher order, but even so it is probably significant. On the other hand, the distinction between licheniformin and subtilin is less marked owing to the relatively low resistances to both these antibiotics demonstrable by the method.

Bactericidal tests. The drawbacks of cross-resistance tests where bacteriostatic activity is measured, led us to use bactericidal tests in the same medium. Since streptomycin is bactericidal in nutrient media (Garrod, 1948), it was anticipated that subtilin and licheniformin might also have this property. If the resistance induced to lethal action of the antibiotics were greater than that induced to bacteriostatic action, the cross-resistance tests would give sharper contrasts. Preliminary tests showed the useful bactericidal concentrations to be 12.5 and 125 units licheniformin $/ \mathrm{ml}$, 20 and $200 \mu \mathrm{g}$. subtilin $/ \mathrm{ml}$., and 12.5 units streptomycin $/ \mathrm{ml}$. Incidentally, on a weight basis, subtilin killed strain $P$ much more slowly than did licheniformin; moreover, the ratio of the concentrations of streptomycin and licheniformin of equal bactericidal potency was the same as the ratio of concentrations of equal bacteriostatic potency, whereas with subtilin and licheniformin the bactericidal ratio was considerably greater than the bacteriostatic ratio. These observations suggest that the action of subtilin and licheniformin differ.

Licheniformin-resistant (LR) and subtilin-resistant (SR) M. phlei, as well as the strain $\mathbf{P}$ from which they were derived, were exposed to licheniformin and to subtilin, with suitable controls. The resistant strains had grown in the presence of 5000 units licheniformin $/ \mathrm{ml}$., and $1000 \mu \mathrm{g}$. subtilin $/ \mathrm{ml}$., in broth agar. The concentrations of licheniformin and subtilin in the flasks were 12.5 units $/ \mathrm{ml}$. and $20 \mu \mathrm{g} . / \mathrm{ml}$., respectively, of Dubos medium. In Fig. $2 \mathrm{~A}-\mathrm{F}$, $\log _{10}$ counts are plotted against time. Points based on mean counts of less than one colony per drop are bracketed, and points based on zero mean counts 


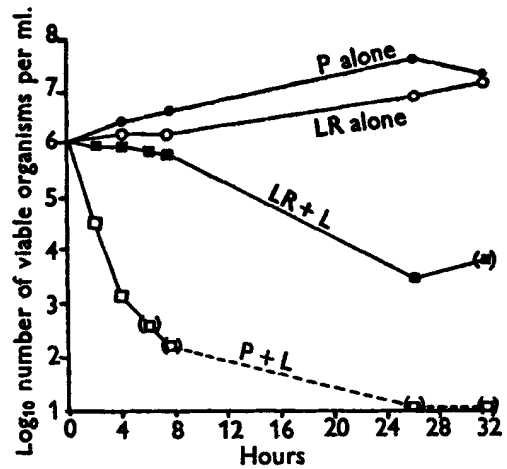

$\mathbf{A}$

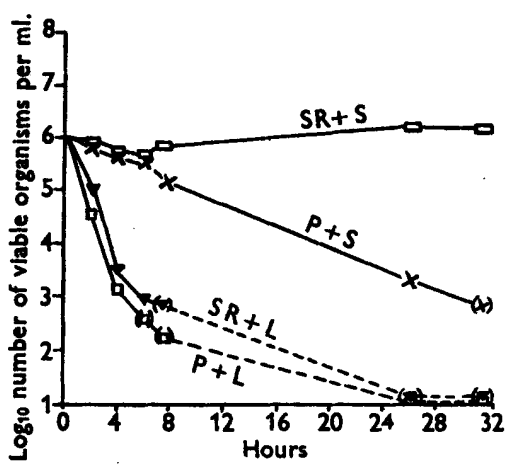

C

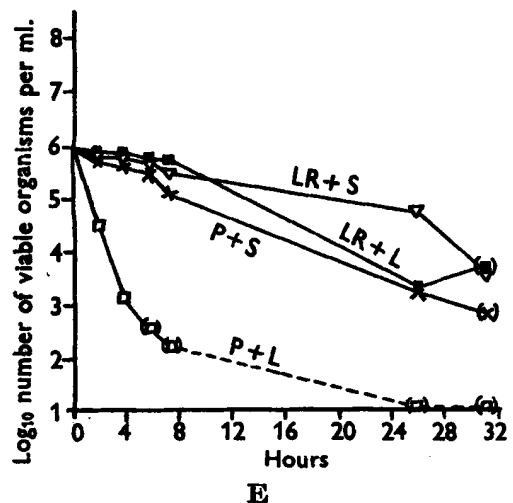

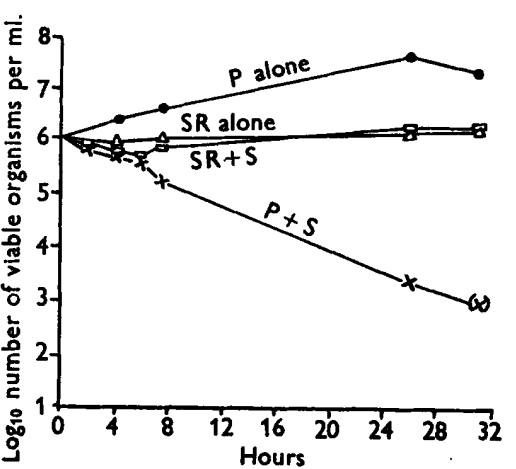

B

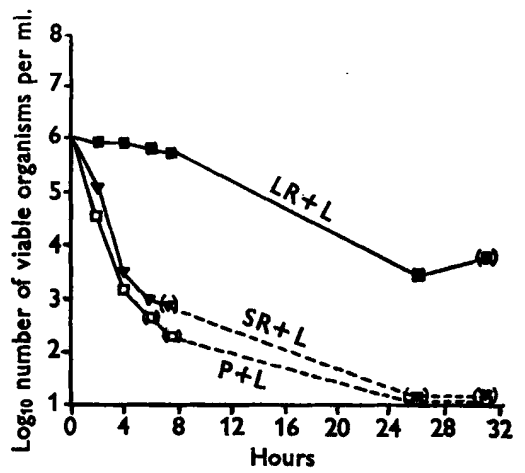

D

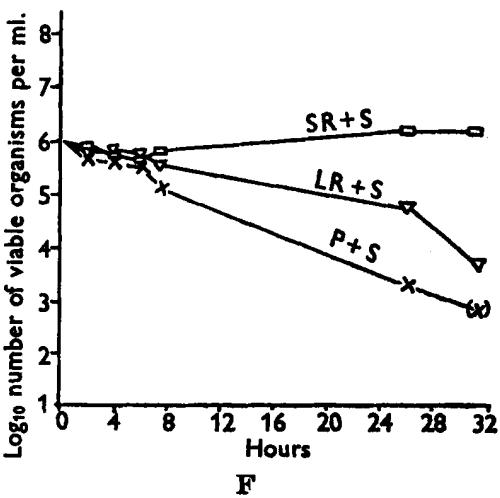

Fig. 2A-F. Viable counts in bactericidal tests of licheniformin and subtilin on induced resistant strains of $M$. phlei in Dubos liquid medium. $L=$ licheniformin; $S=$ subtilin; $\mathbf{P}=$ unmodified strain; $\mathbf{L R}=$ licheniformin-resistant strain; $\mathbf{S R}=$ subtilin-resistant strain. All curves are adjusted to $\mathrm{A} \mathrm{hr}$. reading of $6 \cdot 0$. The initial log counts were in fact: P, 5.7; LR, 6.8; SR, 6.1; P+L, 5.7; LR +L, 6.8; SR +L, 6.0; P +S, 5.8; LR +S, 6.8 ; and $S R+S, 6 \cdot 0$. Brackets indicate readings, and interrupted lines, portions of curves, of doubtful reliability (p. 252). 
per drop of the lowest dilution used (i.e. no colonies from any of the six drops plated from a $10^{-1}$ dilution) are placed arbitrarily on the base-line and conneeted by interrupted lines, to indicate doubtful reliability. The curves have been adjusted, for convenience, to a common starting-point of 6.0 (i.e. initial count of $10^{6}$ viable organisms $/ \mathrm{ml}$.); this was considered justifiable because in a number of tests on each type of mixture, with initial log counts from 4 to 7 as the sole variable, the shape of the curves was found to be similar, and because most initial log counts fell between 5.5 and 6.5. The trends of the curves, rather than a comparison of the individual points on them, are the relevant consideration. Resistance of a modified strain to an antibiotic is indicated by the divergence of the curve for the behaviour of this test-mixture and that for strain $\mathbf{P}$ exposed to the same antibiotic.

The rapid lethal action of licheniformin on strain $P$ is evident (Fig. $2 A$ ); in the strength used, it also killed the LR strain, though the rate was slow in comparison, especially in the first hours. The curves for $\mathbf{P}$ and $\mathbf{L R}$ alone were similar. Subtilin killed P slowly (Fig. 2B), but since the SR strain was hardly affected by this concentration (the curve, after an initial slight fall, rises slowly, rather like the curve for SR alone), a difference in bactericidal effect was manifest. In other experiments the course of the curves for $\mathbf{P}$ and $\mathrm{SR}$ alone was similar. From these observations it is clear that bactericidal tests revealed resistance to the inducing antibiotic in the LR and SR strains used. Larger antibiotic concentrations in other experiments gave no advantage, but, on the contrary, increased the downward gradient of curve $\mathbf{L R}+\mathbf{L}$ more than that of $P+L$ and so brought the two curves nearer together; the effect on curves $\mathbf{S R}+\mathbf{S}$ and $\mathbf{P}+\mathbf{S}$ was similar.

The following contrasts appear from the results of the cross-resistance tests : (1) The SR strain, which was resistant to the lethal effect of subtilin, was almost, if not fully, susceptible to licheniformin, the contrast being shown by the proximity of curves $\mathbf{S R}+\mathbf{L}$ and $\mathbf{P}+\mathbf{L}$ and the divergence of curves $\mathbf{S R}+\mathbf{S}$ and $\mathbf{P}+\mathbf{S}$ (Fig. 2C); this susceptibility also contrasts with the resistance of the LR strain to licheniformin (Fig. 2D). (2) The LR strain, which was resistant to the lethal effect of licheniformin, was also killed rather more slowly than strain $\mathbf{P}$ by subtilin, but comparison of the divergence of curves LR $+S$ and $\mathbf{P}+\mathbf{S}$ with that of $\mathbf{L R}+\mathbf{L}$ and $\mathbf{P}+\mathbf{L}$ shows the cross-resistance of $\mathbf{L R}$ to subtilin to be less than its resistance to the inducing antibiotic (Fig. $2 \mathrm{E}$ ); it is also less than the resistance of the SR strain to its inducing antibiotic (Fig. 2F).

Whether comparison be made of the bactericidal effects of the two antibiotics on the same resistant strain, or of the same antibiotic on the two resistant strains, licheniformin and subtilin can, therefore, be differentiated. There is some evidence of cross-resistance and overlapping activity, but of a minor degree. Other experiments, using the same or ten times the concentrations of antibiotics, supported these conclusions.

Comparison between streptomycin and licheniformin was limited to an experiment in which a streptomycin-resistant strain $(\mathbf{S m R})$ and the unmodified strain $\mathbf{P}$ were exposed to each of these antibiotics. The resistant strain had grown in the presence of 250 units streptomycin/ml. in Dubos medium. The 
concentrations of streptomycin and licheniformin in the flasks were 125 units $/ \mathrm{ml}$. and 12.5 units/ml., respectively. As might be expected, there was a marked divergence between the course of the $\mathrm{SmR}+\mathrm{Sm}$ curve, which, like the curve for SmR alone, rose gradually, and the course of the $\mathbf{P}+\mathrm{Sm}$ curve, which fell rapidly to give zero counts in $6 \mathrm{hr}$; ; streptomycin resistance of the $\mathrm{SmR}$ strain was thus clearly demonstrable. Against this resistance the susceptibility of the SmR strain to licheniformin, shown by the close proximity of curves $\operatorname{SmR}+\mathbf{L}$ and $\mathbf{P}+\mathbf{L}$, stood in strong contrast, thus clearly distinguishing the two antibiotics. The distinction is not complete since no test of the effect of streptomycin on LR bacteria was made.

\section{Production of resistant strains to antibiotic mixtures}

The slight cross-resistance between the licheniformin-resistant and subtilinresistant strains might be accounted for by contamination of one antibiotic with the other. To examine this possibility, resistant strains were induced by mixtures of antibiotics. For technical convenience streptomycin was selected

Table 3. Tests of cross-resistance of strains made resistant by exposures to streptomycin-licheniformin mixtures

\begin{tabular}{|c|c|c|c|c|c|}
\hline \multirow[b]{2}{*}{$\begin{array}{l}\text { Antibiotic used for } \\
\text { inducing resistance }\end{array}$} & \multirow[b]{2}{*}{$\begin{array}{c}\text { No. of } \\
\text { exposures }\end{array}$} & \multicolumn{4}{|c|}{$\begin{array}{l}\text { Antibiotic used for test } \\
\text { (ratio min. inhib. conc. for resistant strains to } \\
\text { value for unmodified strain } P \text { ) }\end{array}$} \\
\hline & & $\begin{array}{l}\text { Streptomycin }+ \\
\text { licheniformin } \\
(1: 1 *)\end{array}$ & $\begin{array}{l}\text { Streptomycin }+ \\
\text { licheniformin } \\
\qquad(9: 1)\end{array}$ & $\begin{array}{l}\text { Licheni- } \\
\text { formin }\end{array}$ & $\begin{array}{l}\text { Strepto- } \\
\text { mycin }\end{array}$ \\
\hline $\begin{array}{l}\text { Streptomycin }+ \\
\text { licheniformin } \\
(1: 1)\end{array}$ & 7 & 4 & $-t$ & 4 & 8 \\
\hline $\begin{array}{l}\text { Streptomycin }+ \\
\text { licheniformin } \\
(9: 1)\end{array}$ & 4 & - & 64 & 4 & $>500$ \\
\hline Licheniformin & $\mathbf{5}$ & - & - & 4 & 2 \\
\hline Streptomycin & 2 & - & - & 1 & $>500$ \\
\hline
\end{tabular}

because it induces resistance easily, and licheniformin because it does so with difficulty. The procedure was one used previously for the single antibiotics: exposure of strain $\mathbf{P}$ to graded concentrations in Dubos medium, with intermediate transfers on egg-yolk agar. Mixtures of streptomycin and licheniformin were prepared, in proportions of $1: 1$ and $9: 1$ by weight (i.e. $1: 10$ and $9: 10$ in units), from two individual solutions at $1 \mathrm{mg} . / \mathrm{ml}$., and were serially diluted as usual. Table 3 records the sensitivities of the resulting strains to the different mixtures, and to their separate components. The strain induced by the $1: 1$ mixture was slightly resistant both to this mixture and to licheniformin (the inhibitory concentration being in each case four times that for strain $\mathbf{P}$ ), and the resistance was similar to that of the LR strain control to licheniformin. Although this strain had a slight resistance to streptomycin (the inhibitory concentration being eight times that for strain $\mathbf{P}$ ), it was much less than that 
of the SmR strain control (a ratio of $>500$ ). The strain induced by the 9:1 mixture also was slightly resistant to licheniformin, though this was considerably less than its resistance to the mixture itself; on the other hand, this strain, like the SmR strain, was highly resistant to streptomycin. Having regard to the susceptibility of the LR strain to streptomycin and of the SmR strain to licheniformin, cross-resistance is thus evident between the strain induced by each antibiotic mixture and that induced by each of the two components singly; in the case of the $1: 1$ mixture the overlap is mainly with licheniformin, and in that of the 9:1 mixture more with streptomycin, though in the latter case even the small amount of licheniformin exerted a detectable effect. Certain combinations of antibiotics, therefore, can, in cross-resistance tests, display the characteristics of one component predominantly and of the other to a minor degree. We recognize that this experiment suffers from being confined to bacteriostatic measurements; no bactericidal tests were made.

\section{DISCUSSION}

The ability of antibiotics to induce resistant strains in bacteria varies greatly. Streptomycin-resistant strains can be produced very easily. It seems more difficult, in general, to produce bacterial cultures resistant to the polypeptide substances derived from the genus Bacillus, at least in vitro, though variation is evident also within this group. Moderate resistance is induced fairly rapidly by subtilin (Salle \& Jann, 1948; and the present work). The same is true for aerosporin and polymyxin with some susceptible bacteria, but with others the increase in resistance is only slight, even after many exposures (Brownlee \& Bushby, 1948; Jawetz \& Coleman, 1949). Our experiments have shown that, in $M$. phlei, licheniformin resistance is somewhat more difficult to induce than subtilin resistance under comparable conditions, both contrasting strongly with streptomycin resistance. Similarly, after subculture of strains isolated from high concentrations of these three antibiotics, streptomycin resistance is stable, while licheniformin-resistant strains partially revert; subtilin resistance is again intermediate. These differences in rate and degree of emergence of resistant strains to licheniformin, subtilin and streptomycin, and in their stability, suggest that these antibiotics are inherently distinct.

Cross-resistance tests in which bactericidal activity is measured by viable counts have proved, in our hands, more precise in the comparison of licheniformin and subtilin than have bacteriostatic tests; and it may be that this technique would be generally useful where the antibiotics whose relationships it is desired to study induce relatively slight degrees of resistance to themselves as judged by reduced bacteriostatic activity. Against the background of more clearly distinguishable resistance to killing, by the inducing antibiotic, bactericidal cross-resistance tests revealed a minor degree of overlap between licheniformin and subtilin, but still indicate substantial differentiation. The distinction is further supported by the much slower rate of killing of the unmodified $M$. phlei by subtilin than by licheniformin. It agrees with salient physical and chemical evidence (Callow et al. 1947). It is more difficult to 
account for the slight cross-resistance. Contamination of one antibiotic by another could produce such an effect, as has been shown here using streptomycin-licheniformin mixtures as a convenient model; but current chemical work on licheniformin offers no support for the hypothesis that this antibiotic contains even small quantities of subtilin (Callow \& Work, 1949). The slight overlap found between licheniformin and subtilin is, therefore, more likely to be a reflexion of a chemical property, e.g. certain amino-acids, in common.

The results of inducing resistance in $M . p h l e i$ to streptomycin-licheniformin mixtures have an incidental additional interest. When the proportion is 9:1 the induced strain has as high a resistance to streptomycin as when the latter is used singly, whereas the strain induced to the 1:1 mixture is only slightly streptomycin resistant. Graessle \& Pietrowski (1949) have shown that $M$. tuberculosis exposed similarly to streptomycin together with $p$-aminosalicylic acid, in a proportion of about $2: 1$, results in delay of the development in vitro of streptomycin resistance-a finding with possible clinical application.

We are indebted to Dr R. K. Callow for the licheniformin and to Dr H. Lineweaver, Western Regional Research Laboratory, California, for the subtilin.

\section{REFERENCES}

Brownlee, G. \& Bushby, S. R. M. (1948). Chemotherapy and pharmacology of aerosporin. Lancet, i, 127.

Callow, R. K., Glover, R. E., Hart, P. D'Arcy \& Hills, G. M. (1947). Licheniformin, an antibiotic substance from Bacillus licheniformis, active against Mycobacterium tuberculosis. Brit. J. exp. Path. 28, 418.

Callow, R. K. \& Work, T. S. (1949). Personal communication.

Duros, R. J. \& Davis, B. D. (1946). Factors affecting the growth of tubercle bacilli in liquid media. J. exp. Med. 83, 409.

Eisman, P. C., Marsh, W. S. \& Mayer, R. L. (1946). Differentiation of antibiotics by resistant strains. Science, 103, 673 .

Fevord, H. L., Drmick, K. P. \& Kuose, A. A. (1948). Isolation of subtilin from submerged cultures. Arch. Biochem. 18, 27.

Frwdes, P. (1931). Medical Research Council's System of Bacteriology in Relation to Medicine, 9, 174. London: H.M. Stationery Office.

Garrod, L. P. (1948). The bactericidal action of streptomycin. Brit. med. J. i, 382.

Graessle, O. E. \& Pietrowski, J. J. (1949). The in vitro effect of para-aminosalicylic acid (PAS) in preventing acquired resistance to streptomycin by Mycobacterium tuberculosis. J. Bact. 57, 459.

Herrold, R. D. (1931). Egg yolk agar medium for the growth of tubercle bacilli. J. infect. Dis. 48, 236.

Jansen, E. F. \& Hirschmann, D. J. (1944). Subtilin-an antibacterial product of Bacillus subtilis: culturing conditions and properties. Arch. Biochem. 4, 297.

$\mathrm{J}_{\text {AWETz, E. \& Coleman, V. R. (1949). Laboratory and clinical observations on }}$ aerosporin (Polymyxin B). J. Lab. clin. Med. 34, 751.

Lewis, J. C. (1947). Chemistry of subtilin. Amer. Ass. for Advancement of Science, Symposium on Antibiotics. Chicago.

LewIS, J. C. (1949). Personal communication.

McIntosh, J. \& SELbIE, F. R. (1943). The production of drug-resistant cultures of bacteria in vitro and a study of their inter-relationships. Brit. J. exp. Path. 24, 246. 
Mrres, A. A. \& Mrsra, S. S. (1938). The estimation of the bactericidal power of the blood. J. Hyg., Camb,, 38, 782.

SAlue, A. J. \& JANN, G. J. (1948). Studies on subtilin-fastness in vitro. J. Bact. 55, 463.

Smrth, D. G. \& Waksman, S. A. (1847). Tuberculostatic and tuberculocidal properties of streptomycin. J. Bact. 54, 253.

Stansly, P. G. (1948). Identification of antibiotics by means of resistant strains of bacteria. J. Bact. 55, 721.

Sulurvan, M., Stahly, G. L., Brrkeland, J. M. \& MYers, W. G. (1946). Reciprocal sensitivities of Staphylococcus aureus to streptomycin, streptothricin and penicillin. Science, 104, 397.

Wien, R., Harrison, J. \& Freeman, W. A. (1948). Diamidines as antibacterial compounds. Brit. J. Pharmacol. \& Chemotherap. 3, 211.

(Received 20 October 1949) 